02

\title{
О прозрачности щелочно-галоидных кристаллов в терагерцовой области спектра
}

\author{
() И.А. Каплунов ${ }^{1}$, Г.И. Кропотов ${ }^{2}$, В.Е. Рогалин ${ }^{3}$, А.А. Шахмин ${ }^{2}$ \\ ${ }^{1}$ Тверской государственный университет, \\ 170100 Тверь, Россия \\ ${ }^{2}$ ООО „Тидекс“, \\ 194292 Санкт-Петербург, Россия \\ ${ }^{3}$ Институт электрофизики и электроэнергетики РАН, \\ 191186 Санкт-Петербург, Россия \\ e-mail: grigorykropotov@tydex.ru, kaplunov.ia@tversu.ru,v-rogalin@mail.ru
}

Поступила в редакцию 07.04.2020 г.

В окончательной редакции 19.05.2020 г.

Принята к публикации 16.06.2020 г.

Исследовано пропускание в терагерцовой (ТГц) области (до $3000 \mu \mathrm{m})$ широко применяемых в инфракрасной области спектра щелочно-галоидных монокристаллов хлористого натрия $(\mathrm{NaCl})$, хлористого калия $(\mathrm{KCl})$, бромистого калия $(\mathrm{KBr})$ и йодистого рубидия $(\mathrm{RbI})$. Получены зависимости коэффициента поглощения этих материалов в диапазоне $0.9-3000 \mu \mathrm{m}$. Вблизи 1000-3000 $\mu \mathrm{m}$ исследованные материалы прозрачны, что позволяет их использовать в ТГц приборах миллиметрового диапазона.

Ключевые слова: щелочно-галоидные кристаллы, поглощение, терагерцовая область.

DOI: $10.21883 /$ OS.2020.10.50017.128-20

\section{Введение}

Терагерцовое (ТГц) излучение занимает промежуточное положение между инфракрасным излучением и радиоволнами и представляет значительный интерес для ряда фундаментальных (химия, физика, астрономия) и прикладных областей. В ТГц области наблюдаются спектры излучения астрономических объектов, а также спектры сложных органических молекул, в том числе таких как взрывчатые, отравляющие и наркотические вещества, вредные вещества - загрязнители атмосферы, а также белки, ДНК. Многие материалы, в том числе текстиль, пластики и биологические ткани прозрачны для ТГц волн. Благодаря низкой энергии фотона ТГц излучение не вызывает повреждений, характерных для ионизирующих излучений [1-3], и поэтому применяется в медицинской диагностике, а также для контроля качества и/или обеспечения безопасности без повреждения материала.

До недавнего времени технологии генерации, преобразования и регистрации ТГц излучения были развиты слабее, чем в соседних диапазонах. Поэтому сложившаяся ситуация даже получила название „терагерцового провала“ (THz gap) [1]. Наличие этой проблемы связано с тем, что частоты колебаний ТГц волн высоки для эффективного использования генераторов радиодиапазона, но малы для применения инфракрасных источников излучения.

Однако в последние годы ведется интенсивное освоение ТГц диапазона $[4,5]$. Найдены полосы пропускания ТГц излучения в атмосфере, хотя и узкие, но вполне пригодные для использования [1-3]. Созданы разнообразные источники ТГц излучения, включая лазерные, на которых уже получены значительные уровни мощности (вплоть до $10^{9} \mathrm{~W}$ в импульсе) [6-11]. Разработаны весьма чувствительные приемники ТГц излучения [1-3]. Тем не менее освоение ТГц диапазона затруднено вследствие значительного дефицита качественных оптических материалов, прозрачных в ТГц области [12-14]. К сожалению, известные на сегодня материалы, прозрачные в ТГц области, обладают значительными недостатками. На этом фоне выделяется сравнительно новый оптический материал - поликристаллический алмаз [15-17]. Но пока стоимость и трудности с обработкой во многих случаях препятствуют его широкому применению.

На практике часто необходимо использовать оптический материал, прозрачный не только в ТГц диапазоне, но и в других областях спектра. Это важно, например, для использования в двухдиапазонных тепловизионных приборах ИК + ТГц области спектра $[18,19]$. Также для оптической накачки ТГц лазеров обычно используют $\mathrm{CO}_{2}$-лазер, излучающий в области 9-11 $\mu \mathrm{m}[8-10]$, и в этом случае зачастую возникает необходимость использовать оптические элементы, прозрачные в обоих диапазонах.

В настоящей работе приводятся результаты исследования пропускания и поглощения в ТГц области щелочно-галоидных монокристаллов (ЩГК) хлористого натрия $(\mathrm{NaCl})$, хлористого калия $(\mathrm{KCl})$, бромистого калия $(\mathrm{KBr})$ и йодистого рубидия $(\mathrm{RbI})$. Работа является продолжением наших предыдущих исследова- 

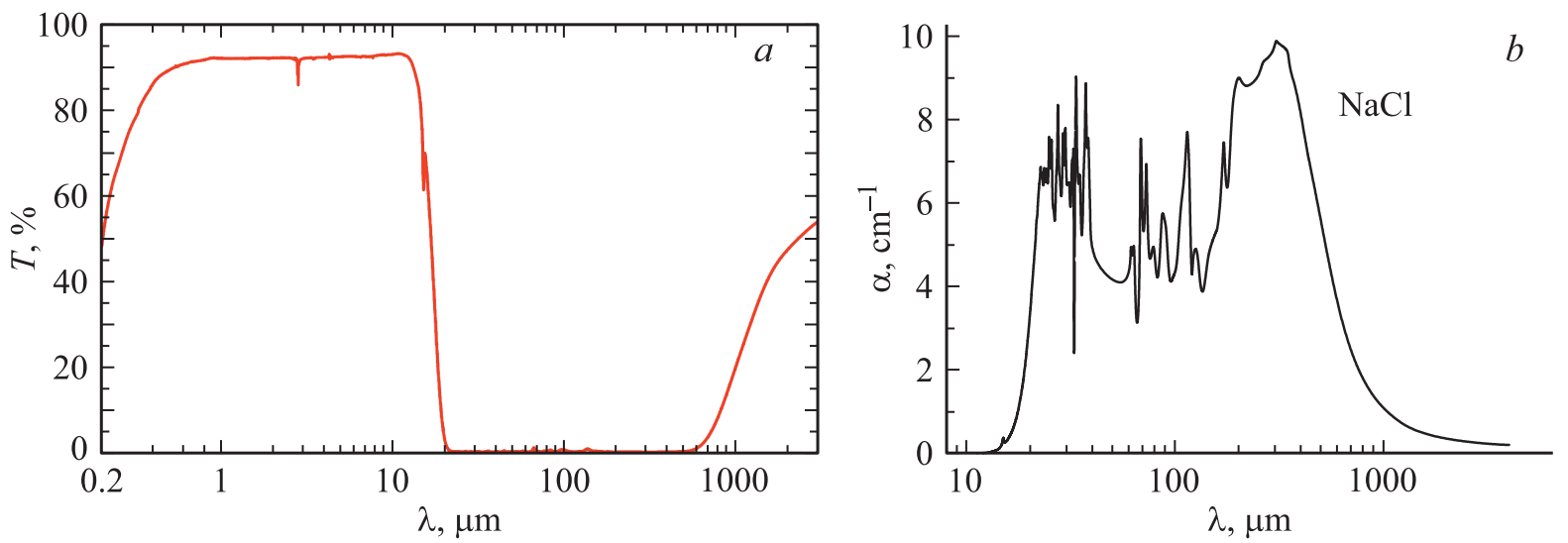

Рис. 1. Спектральная зависимость пропускания $(a)$ и показателя поглощения $(b)$ кристалла $\mathrm{NaCl}$ (толщина $10.15 \mathrm{~mm})$.

ний $[12,20]$, посвященных оптическим материалам для ТГц диапазона.

\section{Материалы и методика эксперимента}

Кристаллы, изученные в настоящей работе, обладают низким показателем преломления, что крайне существенно для применений в ТГц области, где практически исключена возможность просветления оптики традиционными покрытиями [21]. Большие потери на френелевское отражение существенным образом влияют на работу оптических схем. Однако существует возможность частичного просветления оптических деталей для ТГц области. Известно просветление деталей из кремния и кварца полиэтиленовыми и париленовыми покрытиями [22].

Исследованные в работе кристаллы из-за плохих механических свойств чувствительны к условиям эксплуатации: ЩГК - хрупкие, легко раскалывающиеся по спайности (по плоскостям $\langle 100\rangle)$ кристаллы. Они водорастворимы, и работа с ними возможна только в условиях пониженной влажности. В иных случаях для ЩГК используются специальные влагозащитные покрытия [23]. Ранее в ТГц области эти материалы практически не применялись. В ИК технике благодаря хорошим оптическим свойствам ЩГК применяются широко, и для исследований использовались стандартные кристаллы промышленного производства, выращенные из расплава методом Киропулоса в воздушной среде.

Спектральное пропускание регистрировалось с помощью спектрофотометра Photon RT компании Essent Optics, фурье-спектрометра Bruker Vertex 70 в спектральном диапазоне 0.185-670 $\mu \mathrm{m}$. Для Photon RT в диапазоне 185-1700 nm абсолютная погрешность шкалы длин волн составляла $1 \mathrm{~nm}$, для Bruker Vertex 70 погрешность определения волнового числа составляла $0.3-0.5 \mathrm{~cm}^{-1}$. В диапазоне $150-3000 \mu \mathrm{m}$ измерения проводили на приборе TeraK8 MenloSystems. Следует отметить, что этот прибор в диапазоне 150-1500 $\mu \mathrm{m}$

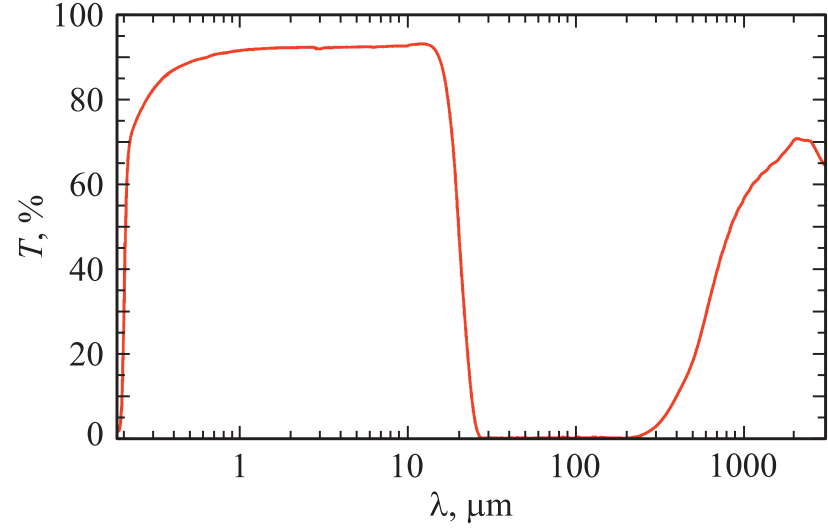

Рис. 2. Спектральная зависимость пропускания кристалла $\mathrm{NaCl}$ (толщина $2 \mathrm{~mm}$ ).

обеспечивает возможность проведения количественных измерений, а в области 1500-3000 $\mu$ т достоверно обеспечиваются лишь качественные измерения. Погрешность в измерении коэффициента пропускания на всех приборах составляла $\sim 0.5 \%$.

Для расчета показателей поглощения (ослабления) $\alpha$ применяли стандартную методику, учитывающую многократные отражения по известной формуле [24,25]. Для определения зависимости показателя преломления от длины волны в рассматриваемых спектральных диапазонах использовали данные [26-29], а также результаты собственных измерений.

Абсолютная погрешность расчетов $\Delta \alpha$ может быть найдена с помощью известного из теории ошибок соотношения:

$$
\Delta f=\sum_{i=1}^{b} \Delta \alpha_{i}\left|f_{x_{i}}^{\prime}\left(a_{1} \ldots a_{k}\right)\right|,
$$

где $\Delta f-$ абсолютная погрешность функции, $a_{i}-i$-й аргумент функции, $\Delta a_{i}$ - абсолютная погрешность этого аргумента. Применительно к формуле для показателей поглощения (ослабления) $\alpha$ получается следующий 

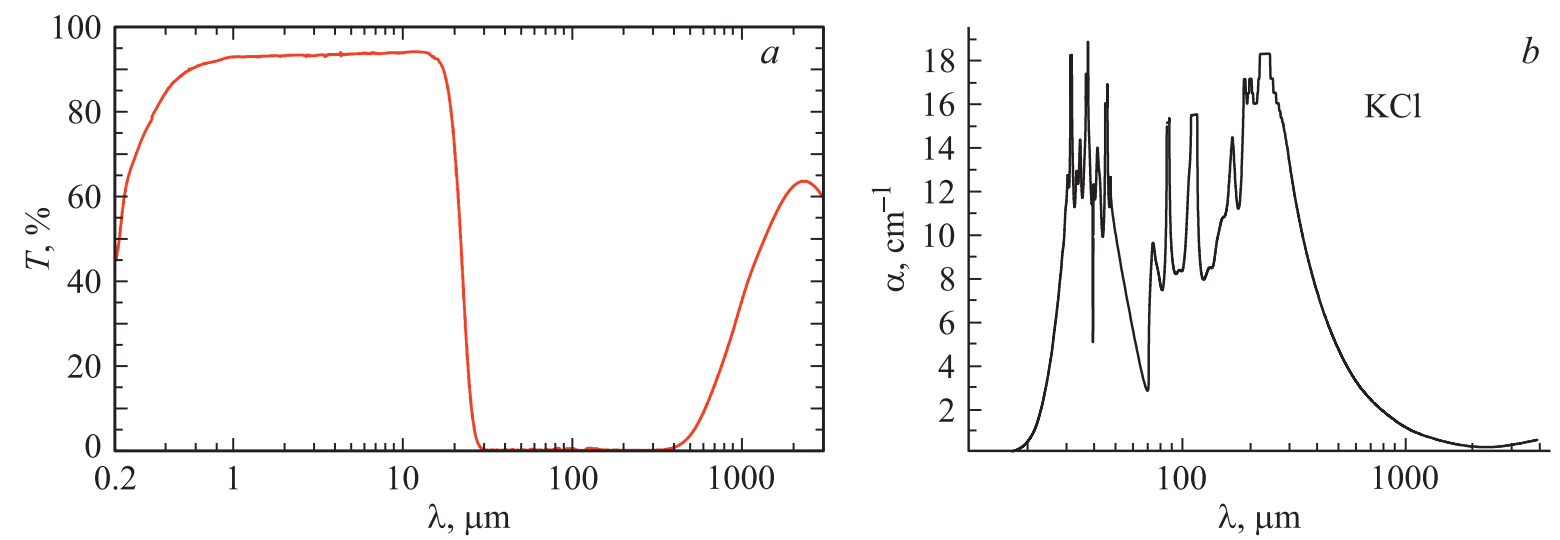

Рис. 3. Спектральная зависимость пропускания $(a)$ и показателя поглощения $(b)$ кристалла $\mathrm{KCl}$ (толщина $6.1 \mathrm{~mm})$.
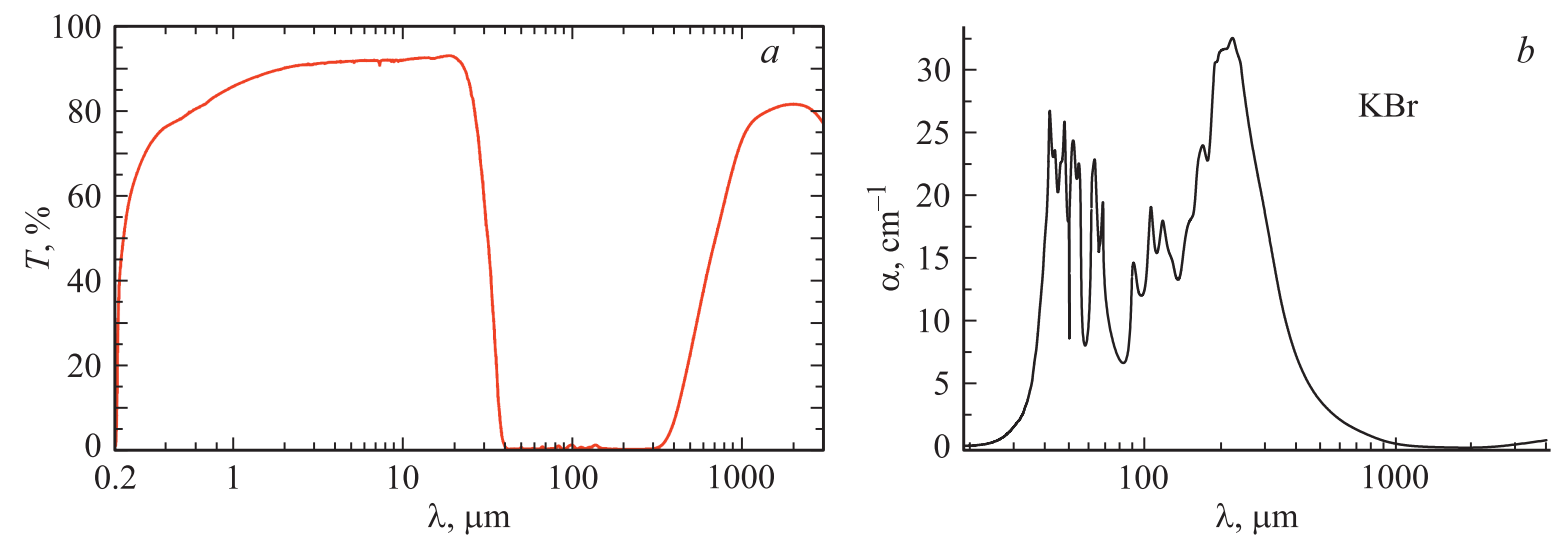

Рис. 4. Спектральная зависимость пропускания (a) и показателя поглощения $(b)$ кристалла $\mathrm{KBr}$ (толщина $3.2 \mathrm{~mm})$.

результат:

$\Delta \alpha=\frac{\Delta h}{h^{2}}\left|\ln \frac{(1-R)^{2}+\sqrt{(1-R)^{4}+4 T^{2} R^{2}}}{2 T}\right|+\frac{2 \Delta R}{h} \times$

$\left|\frac{(1-R) \sqrt{(1-R)^{4}+4 T^{2} R^{2}}+(1-R)^{3}-2 R T^{2}}{\left[(1-R)^{2}+\sqrt{(1-R)^{4}+4 T^{2} R^{2}}\right]\left[\sqrt{(1-R)^{4}+4 T^{2} R^{2}}\right]}\right|$

$+\frac{2 \Delta T}{h T} \times$

$\left.\mid \frac{2 R^{2} T+4 T^{2} R^{2}+(1-R)^{4}}{\left[(1-R)^{2}+\sqrt{(1-R)^{4}+4 T^{2} R^{2}}\right]\left[\sqrt{\left(1-R^{4}+4 T^{2} R^{2}\right)}\right.}\right]$,

где $\Delta R$ и $\Delta T-$ абсолютные погрешности коэффициентов отражения и пропускания, соответственно.

\section{Результаты эксперимента и обсуждение}

Как показывают результаты настоящей работы, галоиды щелочных и щелочно-земельных металлов могут быть использованы и в миллиметровом диапазоне спектра. На рис. 1-5 приведены измеренные спектры пропускания и рассчитанные спектры коэффициентов ослабления для монокристаллов $\mathrm{NaCl}, \mathrm{KCl}, \mathrm{KBr}$ и $\mathrm{RbI}$ от ближнего ИК диапазона до $3000 \mu \mathrm{m}$. Данные по пропусканию в УФ, видимом и ИК диапазонах хорошо известны. В настоящей работе результаты в ИК диапазоне получены на наших образцах и приведены для получения целостной картины прозрачности этой группы материалов в широком диапазоне спектра.

На рис. 1-5 видно, что после полосы пропускания в ИК области во всех описываемых кристаллах наблюдается область интенсивного поглощения, обусловленная фононными процессами [27]. При этом заметно, что край фононного поглощения, как правило, сдвигается в область более длинных длин волн. В области $1000-3000 \mu \mathrm{m}$ эти кристаллы прозрачны и могут быть использованы.

Из известных кристаллов лучшая прозрачность в коротковолновой ТГц области наблюдается в кристаллах RbI (рис. 5). Вообще, следует отметить, что пластинки из кристаллов $\mathrm{RbI}$ прозрачны от $0.24 \mu \mathrm{m}$ до $64 \mu \mathrm{m}$ [28], но они редко применяются в оптике вследствие более высокой, чем у $\mathrm{KBr}$ и CsI, гигроскопичности. В основном данные кристаллы применяются в детекторах частиц высокой энергии [30]. На приведенном на рис. 5 спектре интенсивная полоса поглощения в районе 7-8 $\mu$ m вызвана примесью бария $(\mathrm{Ba})$, введенной 

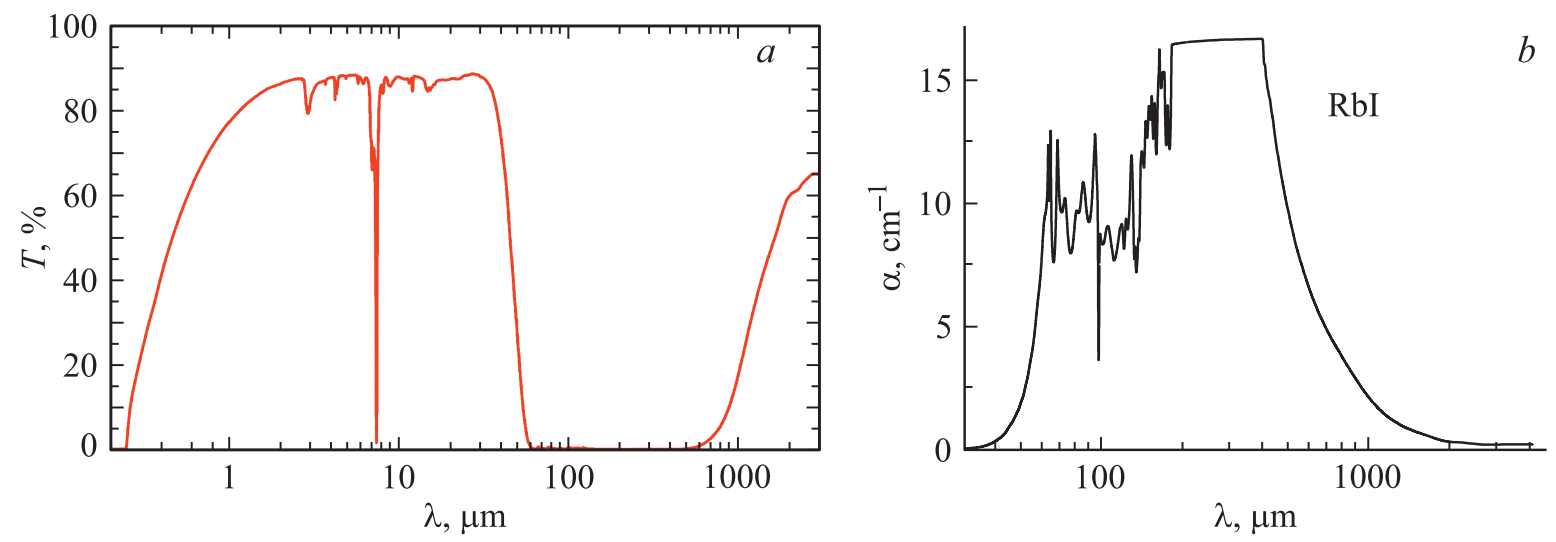

Рис. 5. Спектральная зависимость пропускания $(a)$ и показателя поглощения $(b)$ кристалла RbI (толщина $6.78 \mathrm{~mm})$.

специально для улучшения сцинтилляционных свойств кристалла, которая не влияет на пропускание кристалла в других областях $[28,30,31]$.

К сожалению, как и в других кристаллических материалах [12], в исследованных ЩГК потери пропускания в миллиметровой области заметно выше, чем в ИК области. Это наглядно видно при рассмотрении спектра пропускания кристалла $\mathrm{NaCl}$. На рис 1 приведен спектр пропускания образца толщиной $10.15 \mathrm{~mm}$, а на рис. 2 - образца толщиной $2 \mathrm{~mm}$. Видно, что различная толщина исследованных образцов практически не влияет на величину абсолютного пропускания в ИК области, тогда как в ТГц области даже в максимуме пропускания наблюдается заметное поглощение. Так, в диапазоне $2000 \mu \mathrm{m}$ „тонкий“ образец пропускает 70\% излучения, а „толстый“ образец лишь около $50 \%$. Аналогичные результаты получены и на образцах $\mathrm{KCl}$ и $\mathrm{KBr}$.

\section{Заключение}

В работе измерены спектральные зависимости коэффициента пропускания и рассчитаны спектральные зависимости коэффициента поглощения для щелочногалоидных монокристаллов $\mathrm{NaCl}, \mathrm{KCl}, \mathrm{KBr}$ и $\mathrm{RbI}$ для ТГц области $(30-3000 \mu \mathrm{m})$. Спектральные зависимости для ИК диапазона для данных материалов известны, нами зависимости получены и представлены для сопоставления с результатами для ТГц области, чтобы характеризовать общую картину прозрачности этих материалов в широком диапазоне спектра. Обнаружено, что в миллиметровой области в этих кристаллах наблюдается зона прозрачности, позволяющая использовать эти материалы в приборах этого диапазона.

\section{Финансирование работы}

Работа выполнена с использованием ресурсов предприятия Тидекс и Центра коллективного пользования Тверского государственного университета в рамках государственного задания по научной деятельности.

\section{Конфликт интересов}

Авторы заявляют, что у них нет конфликта интересов.

\section{Список литературы}

[1] Xi-Cheng Zhang, Jingzhou Xu. Introduction to THz Wave Photonics. Springer, 2010. doi 10.1007/978-1-4419-0978-7. Перевод: Си-Чен Чжсан, Джингджю Шю. Терагерцовая фотоника. Москва-Ижевск: ИКИ, 2016. 334 с.

[2] Brundermann E., Hübers H.W., Kimmit M.F. Terahertz Techniques. Heidelberg: Springer, 2012. 383 p.

[3] Lee Y.S. Principles of Terahertz Science and Technology. NY: Springer, 2009. $337 \mathrm{p}$.

[4] Розанов Н.Н., Архипов М.В., Архипов Р.М., Пахомов А.В., Бабушкин И.В. // Опт. и спектр. 2017. Т. 123. № 1. С. 105. doi $10.7868 / \mathrm{S} 0030403417070236$

[5] Розанов Н.Н. // Опт. и спектр. 2009. Т. 107. № 5. С. 761.

[6] Vodopyanov K.L. // Optics Express. 2006. V. 14. N 6. P. 2263.

[7] Веденов А.А., Мыльников Г.Д., Соболенко Д.Н. // УФН. 1982. T. 138. № 3. C. 477.

[8] Tochitsky S.Ya., Chieh Sung, Trubnick S.E., Chan Joshi, Vodopyanov K.L. // J. Opt. Soc. Am. B. 2007. V. 24. N 9. P. 2509.

[9] Tochitsky S.Ya., Ralph J.E., Sung C., Joshi C. // J. Appl. Phys. 2005. V. 98. P. 26101.

[10] Haberberger D., Tochitsky S., Joshi C. // Opt. Express. 2010. V. 18. N 17. P. 17865.

[11] Винокуров Н.А., Шевченко О.А. // УФН. 2018. Т. 188. № 5. C. 493. doi 10.3367/UFNr.2018.02.038311

[12] Рогалин В.Е., Каплунов И.А., Кропотов Г.И. // Опт. и спектр. 2018. Т. 125. № 6. С. 851.

doi 10.21883/OS.2018.12.46951.190-18;

Rogalin V.E., Kaplunov I.A., Kropotov G.I. // Opt. Spectrosc. 2018. V. 125. N 6. P. 1053. doi 10.1134/S0030400X18120172

[13] Parshin V.V. // Int. J. of Infrared and Millimeter Waves. 1994. V. 15. N 2. P. 339.

[14] THz Materials [электронный pecypc]. Режим доступа: http://www.tydexoptics.com/ru/products/thz_optics/ thz_materials/

[15] Ральченко В.Г., Большаков А.П. Углеродная фотоника / Под ред. Конова В.И. М.: Наука, 2017. 327 с. 
[16] Рогалин В.Е., Крымский М.И., Крымский К.М. // Радиотехника и электроника. 2018. Т. 63. № 11. C. 1188. doi 10.1134/S0033849418110098; Rogalin V.E., Krymskii M.I., Krymskii K.M. // J. Commun. Technol. Electron. 2018. V. 63. N 11. P. 1326. doi $10.1134 / \mathrm{S} 1064226918110098$

[17] Рогалин В.Е., Аранчий С.М. // Интеграл. 2012. № 5 (67). C. 7.

[18] Коротаев В.В., Мельников Г.С., Михеев С.В., Самков В.М., Солдатов Ю.И. Основы тепловидения. СПб.: ИТМО, 2012. $123 \mathrm{c}$.

[19] Manolakis D.G., Lockwood R.B., Cooley Th. Hyperspectral Imaging Remote Sensing (Physics, Sensors, and Algorithms). Cambridge Univesity press, 2016. $677 \mathrm{p}$.

[20] Каплунов И.А., Колесников А.И., Кропотов Г.И., Рогалин В.E. // Опт. и спектр. 2019. Т. 126. В. 3. С. 271. doi 10.21883/OS.2019.03.47365.194-18; Kaplunov I.A., Kolesnikov A.I., Kropotov G.I., Rogalin V.E. // Opt. Spectrosc. 2019. V. 126. N 3. P. 191. doi 10.1134/S0030400X19030093

[21] Wheeler J.D., Koopman B., Gallardo P., Maloney P.R., Brugger S., Cortes-Medellin G., Datta R., Darren Dowell C., Glenn J., Golwala S., McKenney C., McMahon J.J., Munson Ch.D., Niemack M., Parshley S., Stacey G. // Millimeter, Submillimeter, and Far-Infrared Detectors and Instrumentation for Astronomy VII / Ed. by Holland W.S., Zmuidzinas J. Proc. SPIE. 2014. V. 9153. P. 91532Z.

[22] [электронный ресурс]. Режим доступа: http://www.tydexoptics.com/ru/products/thz_optics/thz_coatings/

[23] [электронный ресурс]. Режим доступа: http://www.tydexoptics.com/ru/products/coatings/ikpokrytiya/\#ik

[24] Курик М.В. // ЖПС. 1966. Т. 4. № 3. С. 275; Kurik M.V. // J. Appl. Spectrosc. 1966. V. 4. N 3. P. 199.

[25] Каплунов И.А., Колесников А.И., Талызин И.В., Седова Л.В., Шайович С.Л. // Оптический журнал. 2005. Т. 72. № 7. C. 76; Kaplunov I.A., Kolesnikov A.I., Talyzin I.V., Sedova L.V., Shă̌ovich S.L. // J. Opt. Technol. 2005. V. 72. N 7. P. 564.

[26] Li H.H. // J. Phys. Chem. Ref. Data. 1976. V. 5. P. 329. doi 10.1063/1.555536

[27] Mitra S.S. Optical Properties of Highly Transparent Solids. NY.-London, 1975.

[28] Ohlídal Ivan, Franta Daniel. Rubidium Iodide (RbI) Handbook of Optical Constants of Solids. V. III. 1997. P. 857. doi org/10.1016/B978-012544415-6.50136-9

[29] Refractive Index. INFO [электронный ресурс]. Режим доступа: https://refractiveindex.info/about

[30] Meyer F.W., Krause H.F., Vane C.R. // Nucl. Instr. Meth. Phys. Res. B. 2003. V. 205. P. 700. doi org/10.1016/S0168$583 \mathrm{X}(02) 01957-2$

[31] Shunkeyev K., Lushchik A., Myasnikova L., Sagimbaeva Sh., Ubaev Zh., Aimaganbetova Z. // Low Temp. Phys. 2019. V. 45. P. 1127. doi.org/10.1063/1.5125992 